\title{
Horizontal integration of OMIM across the medical school preclinical curriculum for early reinforcement of clinical genetics principles
}

\author{
Adam C. Diehl, BS', Lauren Reader, BS', Ada Hamosh, MD, MPH², Joann N. Bodurtha, MD, MPH²
}

Purpose: With the relentless expansion of genetics into every field of medicine, stronger preclinical and clinical medical student education in genetics is needed. The explosion of genetic information cannot be addressed by simply adding content hours. We proposed that students be provided a tool to access accurate clinical information on genetic conditions and, through this tool, build life-long learning habits to carry them through their medical careers.

Methods: Surveys conducted at the Johns Hopkins University School of Medicine revealed that medical students in all years lacked confidence when approaching genetic conditions and lacked a reliable resource for accurate genetic information. In response, the school created a horizontal thread that stretches across the first-year curriculum and is devoted to teaching students how to use Online

Advances in technology have allowed the field of medical genetics to rapidly expand. Not only are the genetic bases of rare phenotypes being discovered, but the molecular underpinnings of common conditions such as cancer and drug sensitivity are also being characterized and put into clinical use. ${ }^{1-3}$ Database use is central to making this explosion of biomedical information both available and clinically accessible. The Online Mendelian Inheritance in Man (OMIM) database is one particularly useful tool in the practice of medical genetics. OMIM is a full-text resource that draws from accurate primary sources to describe genes and genetic conditions. ${ }^{4}$ OMIM can be searched by clinical features for the generation of differential diagnoses and can link specifically to other databases of genetic information. Thus, OMIM is an excellent gateway for both basic and clinical genetic information, with $\sim 70,000$ unique international users weekly. Medical students need to be trained to function in the growing space in which genetics impacts clinical practice. This need was a primary motivator for the development of the Genes to Society curriculum at Johns Hopkins University School of Medicine (JHU SOM). ${ }^{5}$

As of the 2012-2013 school year, genetics appears in the JHU SOM curriculum as a 5-day anchor course during the first semester of the first year and then as a horizontal strand that pervades the subsequent system-specific blocks of the first- and
Mendelian Inheritance in Man (OMIM) (http://omim.org) and the databases to which it links as a starting point for approaching genetic conditions.

Results: The thread improved the first-year students' confidence in clinical genetics concepts and encouraged use of OMIM as a primary source for genetic information. Most students showed confidence in OMIM as a learning tool and wanted to see the thread repeated in subsequent years.

Conclusion: Incorporating OMIM into the preclinical curriculum improved students' confidence in clinical genetics concepts.

Genet Med advance online publication 17 July 2014

Key Words: clinical genetics; medical education; OMIM

second-year preclinical curriculum. The anchor course involves two to three lectures per day as well as small-group discussions, journal club, and clinical correlations on genetic conditions. The course focuses on basic science concepts central to genetics, such as DNA mutations and repair, chromosome structure and mechanisms, chromosomal anomalies in syndromes and cancer, and current genetic technologies. The horizontal strand takes shape in the form of clinical correlations and lectures on various genetic conditions that are relevant to the subsequent blocks (cardiology, pulmonology, etc). Finally, clinical genetics instruction appears in the last 2 years as an elective clinical clerkship in genetic medicine and as a part of the short introduction to the required pediatrics and women's health core clerkships.

Since its launch in 2009, the Genes to Society curriculum has been in a constant cycle of reevaluation and improvement, and within this cycle, the genetics curriculum figures prominently. Recent reviews of the JHU SOM genetics curriculum revealed that beyond the basic science anchor course in genetics, students received varied and potentially limited instruction on the principles and tools of clinical genetics. The extension of this basic science foundation to the clinical practice of genetics may not be obvious to students and requires further instruction. The horizontal strand that runs through the remainder of 
the preclinical curriculum provides instruction on a few genetic conditions and introduces patient perspectives on these conditions, but it does not adequately integrate the application of clinical genetics. Finally, in the third and fourth years, only a small number of students take the clinical elective in genetic medicine. Therefore, the majority of the class lacks an integrated education in clinical genetics.

This apparent deficit of formal instruction in clinical genetics was substantiated by student responses to their genetics education. An online survey was sent to all 4 years of students, from first-year students who had completed their first year of class to fourth-year students who had recently graduated. From the survey results, it was clear that students wanted a stronger foundation in clinical genetics and that experience with OMIM was lacking. In summary, the review of the current genetics instruction and the results of the survey revealed a "clinical hole" in the genetics curriculum.

To fill this gap under the time constraints of an already full schedule, students needed to be given an engaging tool that could bridge the gap from the basic science foundation that they receive in their first semester to the genetic medicine they will inevitably encounter on any clinical rotation. Students needed (i) a tool to extend basic genetic knowledge to medical genetics practice, (ii) a clinical framework in which to practice using the tool, (iii) a pattern of instruction that reinforced lifelong learning habits, and (iv) a timeline that created confidence in the principles of clinical genetics before the clinical years. With these needs in mind, OMIM was identified as a reliable tool through which the principles of clinical genetics could be taught in an at-home, case-based fashion as a thread within the genetics horizontal strand that runs throughout the preclinical curriculum. The effects of the thread were measured with surveys before and after the intervention.

\section{MATERIALS AND METHODS}

\section{Preintervention survey}

To better characterize the "clinical hole" in the genetics curriculum and how to fill the hole, three online surveys were sent. The first survey was sent to first-year medical students (M1s) who had just finished their inaugural medical school course (anatomy) and were starting their basic science curriculum. To encourage participation, completion of the survey counted toward their grade in the concurrent basic science course. The second survey was sent to second-year medical students (M2s) who had completed their firstsemester genetics course but had not yet entered the wards. The third survey was sent to third- and fourth-year medical students (M3/4s) who had completed their preclinical curriculum and multiple clinical rotations. Using multiplechoice questions and five-point Likert scales, the surveys assessed the students' first source for genetic information, the frequency of searches for this information, the frequency of OMIM use, the type of information OMIM was used to find, confidence in clinical genetics concepts, confidence in OMIM as a tool to learn these concepts, and satisfaction with their education in medical databases as a source of genetic information.

\section{OMIM thread intervention}

The OMIM thread was introduced to M1s concurrently with the start of the student's basic science curriculum, Scientific Foundations of Medicine (SFM), in the first semester after finishing anatomy. After completing the baseline survey, the M1s were introduced to a set of nongraded modules to be completed by the student at home (Supplementary Materials and Methods). These modules presented a clinical situation involving a genetic condition and a set of questions that a clinician might like to answer in this situation (Table 1). In addition to finding clinical information, the modules demonstrated how OMIM can link to basic information about genes and proteins contained in powerful databases such as the UCSC Genome Browser, Ensembl, and the Human Protein Reference Database. After introducing the clinical situation and questions, the module provided step-by-step instructions on how to navigate OMIM and its external links to find the answers to these important clinical questions. In this way, students learned to use OMIM actively and could mold their OMIM training around other more rigid curricular requirements. To practice the process of answering clinically relevant questions about genetic conditions, OMIM was incorporated into the student's Clinical Correlations sessions. During these presentations, a faculty member gives a brief presentation on a medical condition, such as sickle cell anemia, phenylketonuria, and mitochondrial conditions. Then, a patient(s) is invited to speak about their experiences. Students are able to ask the patient specific questions. To incorporate OMIM into these lectures, two slides were shown at the end of the lecture asking students to think about general clinical genetics questions (Table 1), as well as a few more nuanced, patient-centered questions specific to the condition being discussed. Using OMIM, its external links, and their modules as reference, students were given a week to briefly answer these questions in an online Blackboard "quiz" for completion credit. After submitting their responses, students were provided with an answer key showing both the expected answer and the navigation route through OMIM and its external links by which students could find the answer.

Table 1 Clinical questions to ask when encountering a patient with a possible genetic condition What is the differential diagnosis for these signs and symptoms?

What genes are possibly associated with these features?

What allelic variation exists for the gene(s) associated with the patient's condition?

What is the optimal test to confirm the diagnosis? What is its sensitivity?

Are any management guidelines available for this condition?

Are there any drugs/exposures to avoid?

Where can I find a support group for the patient?

Are relatives at risk? Who should be screened?

Are any clinical trials for this condition available? 


\section{Postintervention survey}

At the end of their first semester, having completed their OMIM training modules, three required Clinical Correlations Q\&A sets (on sickle cell disease, phenylketonuria, and mitochondrial disorders), and one optional Q\&A set on hereditary colon cancer, M1s were required to complete a final survey to reassess the same areas as described for the preintervention survey and, in addition, whether they thought the OMIM thread should be repeated.

\section{Statistical methods}

Summary statistics such as the count and percentage of all collected data were presented. For the M1 data, a before-and-after design was used. The intervention effect was assessed by comparing the preintervention evaluation scores with the postintervention evaluation scores. M1 postintervention data were also compared with aggregated data from the M2s and M3/4s. Comparison results ( $P$ values) were derived using Fisher's exact test or the chi-square test to assess binary or categorical data, respectively. Because the surveys were done anonymously, the preintervention and postintervention samples partially overlap but were not able to be linked. Therefore, the test's results are based on the independence assumption.

\section{RESULTS}

\section{Preintervention survey}

The survey sent to the M1s received 118 responses from a class size of 120 . Selected results from the M1s' preintervention survey can be found in Supplementary Table $\mathbf{S 1}$ online. The survey revealed that about $70 \%$ of the class used Google or Wikipedia, $19 \%$ used PubMed, and 0\% used OMIM as their first resource for information on genetic conditions, whereas $9 \%$ had never searched for this type of information before. Moreover, 92\% had never used OMIM before, and most of those who had used it did so less frequently than once per month. In general, students lacked confidence in applying clinical genetics principles, and fewer than 25\% expressed confidence in these skills. However, the majority felt that increased use of OMIM could improve their confidence.

The survey sent to the M2s received 39 responses from a class size of $\sim 120$. The survey revealed that, again, the majority of the class (64\%) used Google or Wikipedia as their first source for genetic information. UpToDate was more popular among M2s (15\%) than M1s (2\%), and OMIM was used by only $5 \%$ of M2s as their first source; $61 \%$ of M2s had never used OMIM before, and most of those who had used it did so less frequently than once per month. M2s also displayed a lack of confidence in applying clinical genetics principles, with fewer than $50 \%$ expressing confidence in any particular skill. They also expressed dissatisfaction with their education on the use of medical databases to find genetic information: $~ 50 \%$ of students were dissatisfied or very dissatisfied students, and only $10 \%$ were satisfied. The majority agreed or strongly agreed that increased use of OMIM during the first 2 years could improve their confidence in clinical genetics skills.
The survey sent to the $\mathrm{M} 3 / 4 \mathrm{~s}$ received 77 responses from a combined class size of $\sim 240$. The most popular first source for genetic information was UpToDate (40\%), with Google (32\%), PubMed (9\%), and Wikipedia (9\%) as the next most popular. Only 2.5\% used OMIM as their first source, $62 \%$ had never used OMIM before, and 70\% said they had not been introduced to OMIM in their first 2 years of medical school. This suggests that from the second year to the third or fourth year there is limited exposure to OMIM on the wards. The majority of those M3/4s that had used OMIM did so less frequently than once per month. Only $20 \%$ of M3/4s felt confident in their ability to generate differential diagnoses including genetic conditions, and only about $30 \%$ felt confident in their ability to share with patients information about genetic conditions. Around $60 \%$ felt confident in their ability to find information on the clinical presentation, diagnostic testing, and management of genetic conditions. Despite this general lack of confidence, $95 \%$ of $\mathrm{M} 3 / 4 \mathrm{~s}$ had seen a patient on the wards with a genetic condition, and about $75 \%$ were including genetic conditions on their differential diagnoses more than infrequently.

Around 50\% of M3/M4s agreed that increased use of OMIM during the first 2 years could improve their confidence in finding information on the clinical presentation, diagnostic testing, and management of genetic conditions, as well as sharing information with patients about genetic conditions. Around $38 \%$ agreed that increased use of OMIM could improve their confidence in generating differential diagnoses that include genetic conditions. Overall, only $18 \%$ were satisfied with their education on the use of medical databases to find genetic information, and $31 \%$ were dissatisfied or very dissatisfied. When asked to comment on how the first 2 years could better instill the principle skills of clinical genetics, many students thought that an introduction to OMIM and incorporation of OMIM into small-group learning could be beneficial. Selected aggregate results from the surveys sent to M2s and M3/4s are shown in Supplementary Table S2 online.

\section{Postintervention survey for M1s}

The postintervention survey sent to the M1s received 80 responses from a class size of 120 , and selected results can be found in Supplementary Table S3 online; significant changes between the M1 pre- and postintervention surveys are shown in Table 2. For the postintervention survey, usage of medical databases was assessed as usage outside of that required for Clinical Correlations assignments. This helps to understand student habits unrestricted by curricular requirements and better reflects lifelong learning routines. The postintervention survey revealed that most M1s (54\%) still chose Google as their first source for genetic information. Furthermore, despite the requirement to use OMIM for their Clinical Correlations assignments, $10 \%$ of M1s reported that they had never used OMIM. However, the percentage of M1s using OMIM as their first source increased from 0 to $25 \%(P<0.001)$, and use of Wikipedia decreased from 22 to $5 \%(P<0.001)$. Moreover, the frequency with which OMIM was used increased, with $42 \%$ of 
Table 2 Significant comparisons between M1 surveys

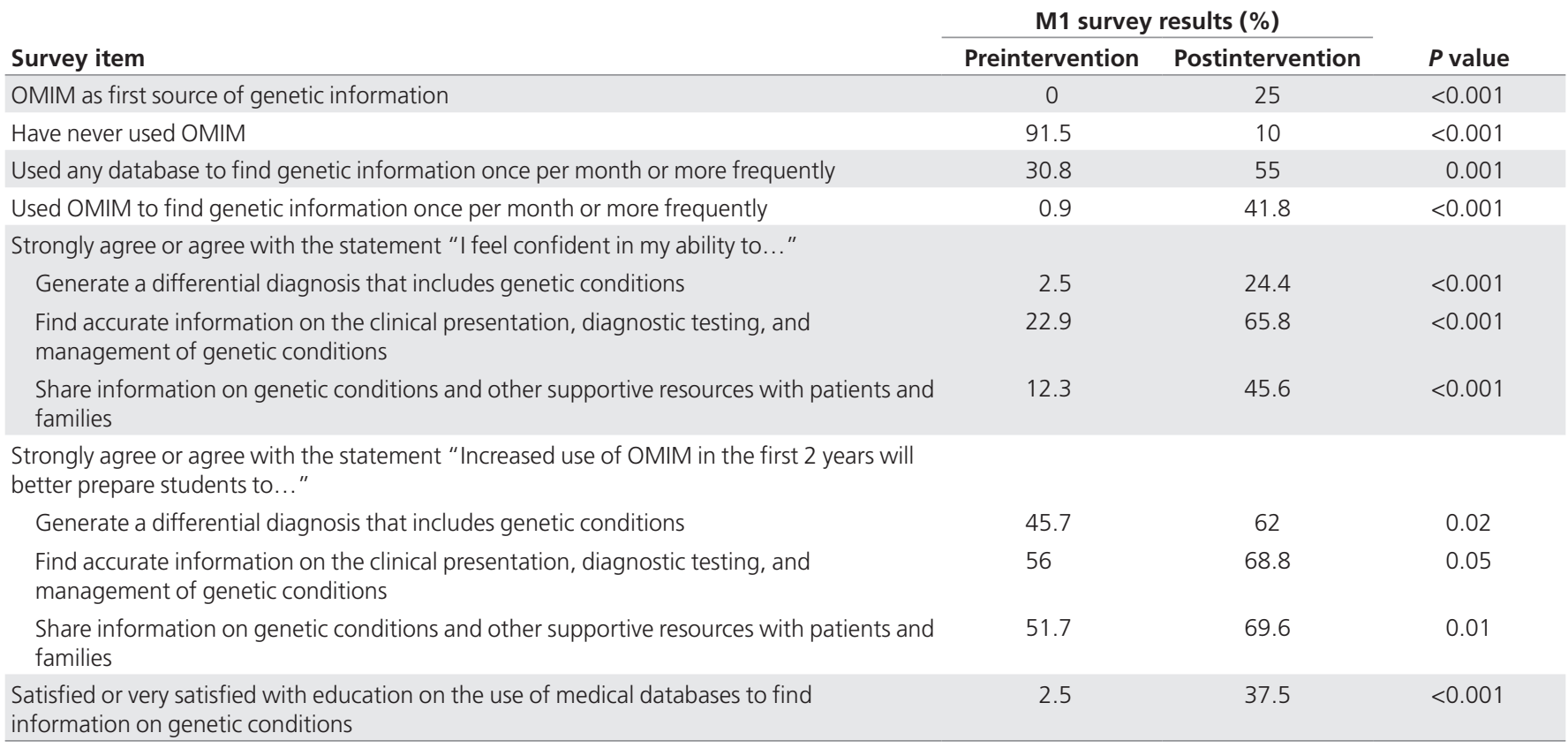

OMIM, Online Mendelian Inheritance in Man.

Table 3 Significant comparisons between M1 postintervention and M2/3/4 surveys

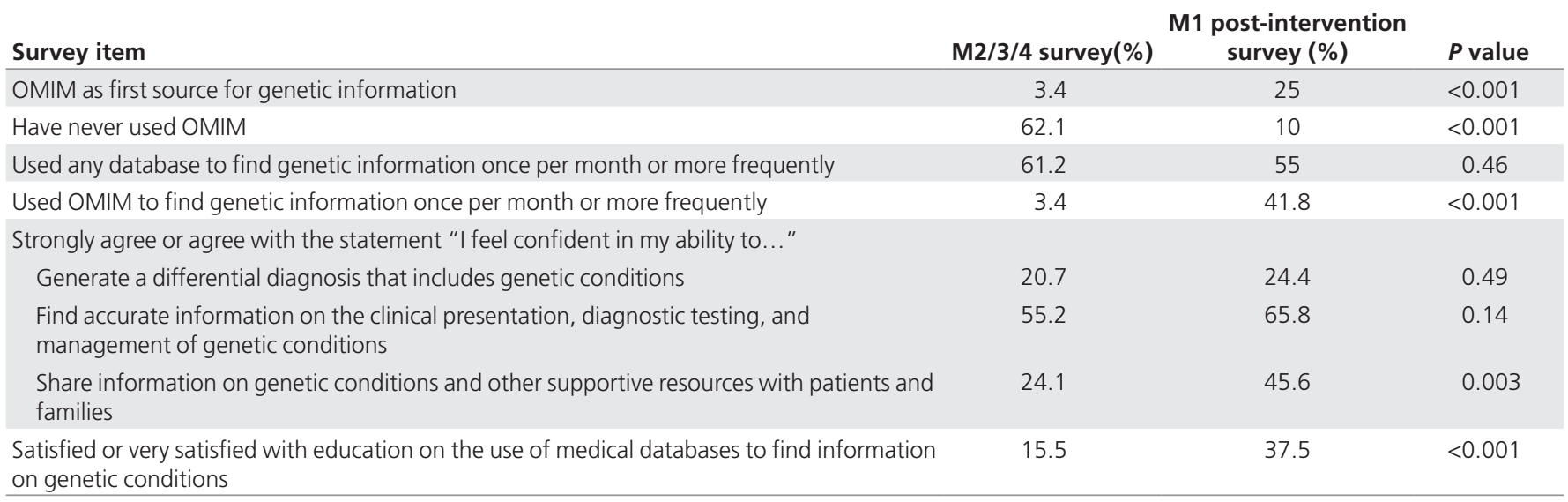

OMIM, Online Mendelian Inheritance in Man.

M1s reporting that they used OMIM at least once per month or more frequently compared with just $1 \%$ before the intervention $(P<0.001)$.

In regard to confidence with clinical genetics principles, a significantly larger percentage of students agreed or strongly agreed that they felt confident in all three domains. In addition, confidence in OMIM as a tool to better educate students in clinical genetic concepts increased in all domains (Table 2). Furthermore, a significantly larger percentage of students were satisfied with their education on medical databases of genetic information, and, when asked whether they thought the OMIM thread should be repeated for next year's class, $66 \%$ of M1s wanted to see it repeated. When asked to comment on their decision, many M1s felt the thread should be repeated because they found OMIM to be a useful learning tool and recognized the increasing importance of clinical genetics. However, many also added that revisions should be made to the thread, such as increased in-class small-group training with OMIM and beginning the thread after the core genetics block had been completed so students could have more familiarity with basic genetics concepts.

Finally, the M1s' postintervention survey results were compared with the aggregate survey results of the M2/3/4s (Table 3 ). Because the M1s concurrently complete the OMIM thread as well as their SFM curriculum, which includes a genetics block, it is likely that the SFM curriculum contributes to differences between the M1s' pre- and postintervention survey results. However, the upperclassmen completed the SFM curriculum without the OMIM thread and can therefore serve as controls that may help isolate the effect of the OMIM thread. 
The upperclassmen also completed additional coursework outside of SFM with variable emphasis on clinical genetics concepts. Thus, the upperclassmen roughly embody the state of clinical genetics education at JHU SOM before the introduction of the OMIM thread. Table 3 shows that OMIM use as a first resource and frequency of OMIM use among the M1s after the intervention was significantly greater than that of the upperclassmen. In addition, the types of information that M1s used OMIM to find were more diverse relative to upperclassmen who use OMIM (Supplementary Table S4 online). In regard to confidence with clinical genetics concepts, a greater percentage of M1s displayed confidence for all three domains compared with upperclassmen (Table 3). However, this finding was statistically significant only for the domain focused on sharing genetic information with patients and families. Finally, $37.5 \%$ of M1s reported being satisfied or very satisfied with their education in the use of medical databases to find genetic information compared with only $15.5 \%$ of upperclassmen.

\section{DISCUSSION}

This report describes the response to a need for early reinforcement of clinical genetics principles that was identified within the JHU SOM preclinical curriculum. This need was further validated by the lack of confidence in these principles displayed by upperclassmen, suggesting that these principles were not reinforced sufficiently in clinical clerkships. Implementation of the thread resulted in an increased number of M1s using OMIM as their primary genetic information resource, with increased confidence in clinical genetic concepts.

In this era of advances in human genome sequencing, pharmacogenomics, and targeted therapeutics, all bolstering public interest in direct-to-consumer testing, the increasing need for education in genetics at all levels of training is well recognized. ${ }^{6}$ Genetics is affecting a wider breadth of medical specialties, and practicing physicians have been found to rely on their medical school courses as their main source of genetics knowledge. ${ }^{7}$ Thus, it is imperative that genetics education be strengthened in the years of medical school before specialty differentiation. Moreover, genetics education must seek to bridge the gap between basic genetics and clinical practice and should show that genetics is practical, affects common conditions, and can be used on a daily basis to improve patient outcomes. ${ }^{8}$ Efforts to meet these objectives are varied, including optional 4-year educational tracks in genetics, ${ }^{9}$ case-based sessions and patientcentered lectures ${ }^{10}$ elective courses on personal genotyping, ${ }^{11,12}$ and virtual cases on genetic testing strategies. ${ }^{13}$

As in this report, multiple schools have recognized the importance of the medical database as a tool for teaching genetics. One of the earliest efforts was undertaken at the Medical College of Virginia in 1986, where each student, as part of their basic genetics coursework, was required to update an entry of McKusick's Mendelian Inheritance in Man, the forerunner of OMIM, by searching the primary literature for new information on the condition, with an emphasis on information of clinical relevance. ${ }^{14}$ In 2001, Washington University School of
Medicine developed a solitary module to teach students to use National Center for Biotechnology Information tools such as OMIM, PubMed, and BLAST. ${ }^{15}$ They prompted students with clinical vignettes and DNA sequence fragments, and students were then required to use these tools to identify the disease and disease gene. This initiative focused on the principles of genomics and its molecular fundamentals but was extended in a more clinical direction at the Pritzker School of Medicine in 2006. At Pritzker, two computer-based training sessions were developed: one for the first year and one for the third year of medical school. ${ }^{16}$ Both sessions required students to use databases (such as BLAST, Entrez, PubMed, OMIM, GeneTests, and Genetic Alliance) to answer problem sets. The first-year session was modeled on the Washington University School of Medicine module and emphasized basic genetic principles but also asked students to find treatment options for identified diseases. The third-year session was designed to introduce students to Internet resources, especially OMIM and linked databases such as GeneTests and Genetic Alliance, that could assist in evaluating, diagnosing, and counseling patients with genetic conditions. Clearly, multiple medical schools are facing the need to teach clinical genetics concepts as well as the need for tools that make these concepts accessible.

JHU SOM's experience in early integration of clinical genetics principles adds to the insights drawn by these other initiatives and provides a practical model for integration at other institutions. The OMIM thread was structured around a number of principles to promote effective learning while minimizing content hours. First, students were given a tool that provides reliable and accurate information while reinforcing clinical genetics principles. A tool allows students to teach themselves genetics on the fly, a pattern of learning they will need to adopt as busy clinicians trying to keep up with the ever-changing field of genetics. In fact, a 2004 report on genetics education from the Association of American Medical Colleges' Medical School Objectives project states that students should learn the clinical application of genetics through consulting primary literature and online databases. ${ }^{17}$ Second, students practiced using OMIM longitudinally, outside of the classroom and in association with clinical encounters. This method models and promotes the style of lifelong learning required of a clinician seeing a patient with a genetic condition that may be new to the clinician. Finally, the thread was designed to be easily incorporated into the preclinical curriculum, providing an early introduction to clinical genetic concepts and preparing students for their clinical years. The thread requires minimal additions to in-class time; the modules and clinical correlations exercises are done at home over the course of a week. It can also easily extend across discrete subject blocks because genetic conditions are present in every block, allowing the association of OMIM exercises with lectures or patient-based Clinical Correlations on these conditions.

This study has a number of limitations. First, although the M1s' preintervention survey response rate was close to $100 \%$, only two-thirds of the class responded to the postintervention 
survey. Nonrespondents might have had an even lower use of OMIM. Moreover, only about one-third of upperclassmen were represented by their survey. Second, the upperclassmen do not act as a perfect control group. Although they completed the SFM course without the OMIM thread, they also bring to their survey answers perspectives that reflect their closer contact with patients, such as the difficulties of sharing information with patients. The third limitation was reinforcement of training. A number of students did not complete the modules and subsequently found it frustrating to answer their Clinical Correlations question sets using OMIM. This generated a number of requests for in-class training with OMIM. Finally, although our intervention encouraged greater use of OMIM, only $25 \%$ of M1s report using OMIM as their first source for genetic information, with Google still predominating. It is likely that further training with OMIM is required for its ease of answering clinical questions to exceed that of a Google search.

Moving forward, JHU SOM is currently working to incorporate OMIM-centered, case-based exercises in clinical genetics throughout the rest of the Genes to Society curriculum in association with block-specific genetic conditions. Although some cursory training in databases linked to OMIM was provided in the modules, JHU SOM is developing more extensive training in genomics tools through a clinical sequencing elective that is in development. Further, JHU SOM recognizes the diversity of learning styles among medical students and the importance of teaching by example. Thus, JHU SOM hopes to develop a smallgroup session led by medical genetics faculty that uses a real clinical case and illustrates how a clinician would use medical databases in practice. Finally, JHU SOM is in the process of creating an intersession course for M3/4s that provides in-depth training in the essentials of clinical genetics.

\section{SUPPLEMENTARY MATERIAL}

Supplementary material is linked to the online version of the paper at http://www.nature.com/gim

\section{ACKNOWLEDGMENTS}

We thank all the medical students for completing the surveys and question sets, Patricia Thomas and all the SFM faculty for the time they invest in curricular improvement, including this study, and
Susan Mrozowski and Theo Karpovich for all their administrative help.

\section{DISCLOSURE}

The authors declare no conflict of interest.

\section{REFERENCES}

1. Lennard L. Implementation of TPMT testing. Br J Clin Pharmaco/ 2014;77:704714.

2. Carlson JJ, Roth JA. The impact of the Oncotype Dx breast cancer assay in clinical practice: a systematic review and meta-analysis. Breast Cancer Res Treat 2013;141:13-22.

3. Evans JP, Khoury MJ. The arrival of genomic medicine to the clinic is only the beginning of the journey. Genet Med 2013;15:268-269.

4. Amberger J, Bocchini CA, Scott AF, Hamosh A. McKusick's online Mendelian inheritance in man (OMIM®). Nucleic Acids Res 2009;27:793-796.

5. Wiener CM, Thomas PA, Goodspeed E, Valle D, Nichols DG. "Genes to society"-the logic and process of the new curriculum for the Johns Hopkins University School of Medicine. Acad Med 2010;85:498-506.

6. Demmer LA, Waggoner DJ. Professional medical education and genomics. Annu Rev Genomics Hum Genet 2014; e-pub ahead of print 12 March 2014.

7. Hunter A, Wright P, Cappelli M, Kasaboski A, Surh L. Physician knowledge and attitudes towards molecular genetic (DNA) testing of their patients. Clin Genet 1998;53:447-455.

8. Guttmacher AE, Porteous ME, Mclnerney JD. Educating health-care professionals about genetics and genomics. Nat Rev Genet 2007;8:151-157.

9. Dhar SU, Alford RL, Nelson EA, Potocki L. Enhancing exposure to genetics and genomics through an innovative medical school curriculum. Genet Med 2012;14:163-167.

10. Korf BR. Integration of genetics into clinical teaching in medical school education. Genet Med 2002;4(suppl 6):33S-38S.

11. Vernez SL, Salari K, Ormond KE, Lee SS. Personal genome testing in medical education: student experiences with genotyping in the classroom. Genome Med 2013;5:24

12. Walt DR, Kuhlik A, Epstein SK, et al. Lessons learned from the introduction of personalized genotyping into a medical school curriculum. Genet Med 2011;13:63-66

13. Bean LJ, Fridovich-Keil J, Hegde M, Rudd MK, Garber KB. The virtual diagnostic laboratory: a new way of teaching undergraduate medical students about genetic testing. Genet Med 2011;13:973-977.

14. Bodurtha JN, Townsend JI, Proud VK, Nance WE. Updating McKusick: an educational exercise for medical students. Am J Med Genet 1986;24:505-511.

15. Magee J, Gordon J, Whelan A. Bringing the human genome and the revolution in bioinformatics to the medical school classroom: a case report from Washington University School of Medicine. Acad Med 2001;76:852-855.

16. Waggoner DJ, Martin CL. Integration of internet-based genetic databases into the medical school pre-clinical and clinical curriculum. Genet Med 2006;8:379-382.

17. Assoc. Am. Med. Coll. Contemporary issues in medicine: genetics education. Report VI, Contemporary Issues in Medicine: Genetics Education, Medical School Objectives Project. Assoc. Am. Med. Coll. Chicago, June 2004. http://members.aamc.org/eweb/upload/Contemporary $\% 20$ Issues $\% 20$ in $\% 20$ Med $\% 20$ Genetics\%20Education\%20Report\%20VI.pdf Accessed 4 June, 2014. 